\title{
Extra Dirac points in the energy spectrum for superlattices on single-layer graphene
}

\author{
M. Barbier, ${ }^{1}$ P. Vasilopoulos, ${ }^{2}$ and F. M. Peeters ${ }^{1}$ \\ ${ }^{1}$ Department of Physics, University of Antwerp, Groenenborgerlaan 171, B-2020 Antwerpen, Belgium \\ ${ }^{2}$ Department of Physics, Concordia University, 7141 Sherbrooke Ouest, Montréal, Quebec, Canada H4B 1R6 \\ (Received 14 September 2009; revised manuscript received 9 December 2009; published 25 February 2010) \\ We investigate the emergence of extra Dirac points in the electronic structure of a periodically spaced barrier \\ system, i.e., a superlattice, on single-layer graphene, using a Dirac-type Hamiltonian. Using square barriers \\ allows us to find analytic expressions for the occurrence and location of these new Dirac points in $\mathbf{k}$ space and \\ for the renormalization of the electron velocity near them in the low-energy range. In the general case of \\ unequal barrier and well widths the new Dirac points move away from the Fermi level and for given heights \\ of the potential barriers there is a minimum and maximum barrier width outside of which the new Dirac points \\ disappear. The effect of these extra Dirac points on the density of states and on the conductivity is investigated.
}

DOI: 10.1103/PhysRevB.81.075438

PACS number(s): 71.10.Pm, 73.21.-b, 81.05.U-

\section{INTRODUCTION}

Graphene, a one-atom thick layer of carbon atoms, has been a topic of intense study since its experimental realization $^{1}$ in 2004. Interest in graphene results, in particular, from the prediction that carriers in it behave as massless, chiral Dirac fermions, moving in a two-dimensional (2D) plane and described by a Dirac-type Hamiltonian. This model predicts unusual electronic properties such as the gapless electronic spectrum, the perfect transmission at normal incidence through any potential barrier, i.e., the Klein paradox $^{2,3}$ which was recently addressed experimentally, ${ }^{4}$ the zitterbewegung recently verified, ${ }^{5}$ etc., see Ref. 6 for a recent review.

Motivated by all these properties, condensed matter physicists started extending the known properties of a twodimensional electron gas (2DEG) in semiconductor materials to the relativistic 2D fermions (Dirac electrons) in graphene. Another particularly interesting system to consider is the application of a periodic potential to graphene, that is, a superlattice (SL), which under special conditions leads to collimation of electron beams. ${ }^{7-9}$

As found recently in Ref. 10, using a tight-binding formalism, the dispersion relation for such a SL can reveal extra Dirac points at the Fermi level. ${ }^{11}$ Close to the Fermi level the electronic properties of graphene are well described by the massless 2D Dirac equation. In two recent studies, Refs. 12 and 13, an exact condition was found for the emergence of extra Dirac points (zero modes) in the presence of a sinusoidal or square-wave SL potential. However, both studies are not able to describe the character and spatial distribution in $\mathbf{k}$ space of these new Dirac points, as they expand the spectrum for small $k_{y}$. In this work we describe under which condition this is possible and also where these extra Dirac points arise in the electronic structure of massless Dirac fermions in single-layer graphene when a square-wave periodic potential is applied. Further, we analytically investigate the anisotropic renormalization of the group velocities at these new Dirac points, and find that the degree of the renormalization depends on the parameter $u \propto V_{0} L$, which is linear in the barrier height $V_{0}$ and period $L$ of the SL, in the sense that, $v_{x}>v_{y}$ holds for $u$ such that an extra Dirac point arises while for very high values $u$ we have $v_{x}<v_{y}$. Moreover, we also consider the case of unequal barrier and well widths, not treated previously, that results in a qualitatively different electronic spectrum.

We organize the paper as follows. In Sec. II we introduce our model. In Sec. III we investigate the emergence of the extra Dirac points, approximate the implicit dispersion relation for small energies, and take a closer look at the group velocity near the extra Dirac points. Further we investigate the influence of the features of the spectrum on the density of states and conductivity. Finally, we make a summary and concluding remarks in Sec. IV.

\section{MODEL}

We describe the electronic structure of an infinitely large flat graphene flake by the nearest-neighbor, tight-binding model and consider solutions with energy and wave vector close to the $\mathrm{K}$ point. The relevant Hamiltonian is $\mathcal{H}$ $=v_{F} \vec{\sigma} \cdot \hat{\vec{p}}+1 \mathrm{~V}$, with 1 the $2 \times 2$ unit matrix. Explicitly $\mathcal{H}$ is given by

$$
\mathcal{H}=\left(\begin{array}{cc}
V & -i v_{F} \hbar\left(\partial_{x}-i \partial_{y}\right) \\
-i v_{F} \hbar\left(\partial_{x}+i \partial_{y}\right) & V
\end{array}\right)
$$

where $\vec{p}$ is the momentum operator and $v_{F} \approx 10^{6} \mathrm{~m} / \mathrm{s}$ the Fermi velocity. In the presence of a one-dimensional (1D) square-wave potential $V(x)$, such as the one shown in Fig. 1, the equation $(\mathcal{H}-E) \psi=0$ admits solutions of the form $\psi(x) e^{i k_{y} y}$ with

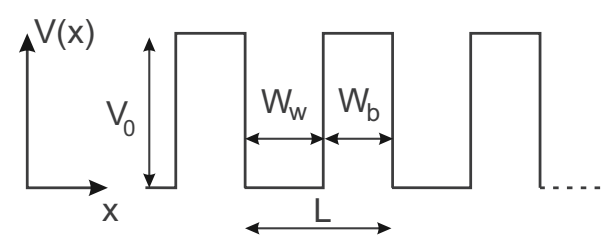

FIG. 1. Schematics of the superlattice potential $V(x)$ consisting of square barriers. 


$$
\psi(x)=\left(\begin{array}{c}
1 \\
s e^{i \phi}
\end{array}\right) e^{i \lambda x}, \quad \psi(x)=\left(\begin{array}{c}
1 \\
-s e^{-i \phi}
\end{array}\right) e^{-i \lambda x},
$$

$\lambda=\left[(\varepsilon-u(x))^{2}-k_{y}^{2}\right]^{1 / 2}, \quad \tan \phi=k_{y} / \lambda, \quad s=\operatorname{sgn}[\varepsilon-u(x)]$, $\varepsilon=E / v_{F} \hbar$, and $u(x)=V(x) / v_{F} \hbar$; the parameters $\varepsilon$ and $u(x)$ are in units of inverse length.

\section{Square-barrier superlattice}

We consider an infinite number of periodically spaced barriers, as shown in Fig. 1, with unit cell length $L$ and barrier (well) width $W_{b}\left(W_{w}\right)$. It is convenient to introduce the dimensionless variables $\varepsilon \rightarrow \varepsilon L, \quad k_{y} \rightarrow k_{y} L$, $k_{x} \rightarrow k_{x} L, \quad u \rightarrow u_{0} L=V_{0} L / v_{F} \hbar, \quad x \rightarrow x / L, \quad W_{b} \rightarrow W_{b} / L, \quad$ and $W_{w} \rightarrow 1-W_{b} / L$. The wave function of this periodic system is a Bloch function and the transfer matrix $\mathcal{T}$ pertinent to it leads to an expression for the dispersion relation, see Appendix A. For $\left|k_{y}\right|<\left|\varepsilon_{w}\right|$ and $\varepsilon_{w}$ as in Eq. (6), the transfer matrix $\mathcal{T}$ can be written as ${ }^{14}$

$$
\mathcal{T}=\left(\begin{array}{cc}
w & z \\
z^{*} & w^{*}
\end{array}\right)
$$

then the dispersion relation becomes

$$
\cos \left(k_{x}\right)=\mathfrak{R}\left\{e^{-i \lambda} w\right\},
$$

with $w$ given by

$$
w=e^{i \lambda W_{b}\left[\cos \Lambda W_{b}-i G \sin \Lambda W_{b}\right]}
$$

and

$$
\begin{gathered}
\varepsilon_{w}=\varepsilon+u W_{b}, \quad \varepsilon_{b}=\varepsilon-u W_{w}, \quad G=\left(\varepsilon_{w} \varepsilon_{b}-k_{y}^{2}\right) / \lambda \Lambda, \\
\lambda=\left[\varepsilon_{w}^{2}-k_{y}^{2}\right]^{1 / 2}, \quad \Lambda=\left[\varepsilon_{b}^{2}-k_{y}^{2}\right]^{1 / 2} .
\end{gathered}
$$

Writing the rhs of Eq. (4) explicitly gives

$$
\cos k_{x}=\cos \lambda W_{w} \cos \Lambda W_{b}-G \sin \lambda W_{w} \sin \Lambda W_{b} .
$$

Although this derivation is only correct for $\left|k_{y}\right|<\left|\varepsilon_{w}\right|$, Eq. (7) is also valid beyond this limitation, see Ref. 15. From Eq. (7) it can be seen that the dispersion relation possesses the symmetry property $\varepsilon \rightarrow-\varepsilon$ for $W_{b} \leftrightarrow W_{w}$. The asymmetric spectrum is not unexpected because the symmetry of the potential about the Fermi level is lost for $W_{b} \neq 1 / 2$. For $W_{b}$ $=1 / 2$ we have

$$
\cos k_{x}=\cos \frac{\lambda}{2} \cos \frac{\Lambda}{2}-G \sin \frac{\lambda}{2} \sin \frac{\Lambda}{2},
$$

where $\varepsilon_{w}=\varepsilon+u / 2$ and $\varepsilon_{b}=\varepsilon-u / 2$. For this interesting case, the potential possesses particle-hole symmetry and the extra Dirac points originate at the Fermi level; we will show their arrangement, in $\mathbf{k}$ space, in Sec. III A.

In Fig. 2(a) the spectrum resulting from Eq. (8) for equal barrier and well widths, i.e., for $W_{w}=W_{b}=1 / 2$, is plotted for $u=10 \pi$. As can be seen, the spectrum is symmetric about the Fermi level; there are two extra Dirac points on both sides of the main Dirac point, and their velocities are renormalized. The anisotropic behavior of the new Dirac cones is clearer in the projection of the conduction band shown in Fig. 2(b).
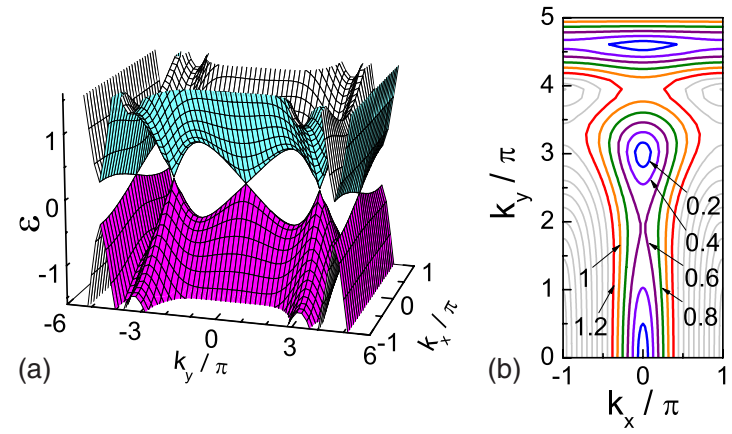

FIG. 2. (Color online) (a) Valence and conduction bands of the spectrum of a SL with square barriers of width $W_{b}=1 / 2$ and height $u=10 \pi$. (b) Contour plot of the conduction band.

Further details about the renormalization of the velocities will be given in Sec. III C.

For unequal barrier and well widths the spectrum is shown in Fig. 3(a) for $W_{b}=1-W_{w}=0.4$ and $u=6 \pi$. The spectrum is no longer symmetric about the Fermi level, the two extra Dirac points are shifted in energy relative to the main point and their velocities are renormalized. The location of the extra Dirac points will be investigated in Sec. III.

\section{ELECTRONIC STRUCTURE}

\section{A. Appearance of extra Dirac points}

In order to find the location of the Dirac points we assume $k_{x}=0, \varepsilon=0$, and $W_{b}=W_{w}=1 / 2$ in Eq. (8). Then Eq. (8) becomes

$$
1=\cos ^{2} \lambda / 2+\left[\left(u^{2} / 4+k_{y}^{2}\right) /\left(u^{2} / 4-k_{y}^{2}\right)\right] \sin ^{2} \lambda / 2,
$$

which has solutions for $u^{2} / 4-k_{y}^{2}=u^{2} / 4+k_{y}^{2}$ or $\sin ^{2} \lambda / 2=0$. For the first possibility $k_{y}=0$ is the only solution and corresponds to the usual Dirac point. The second possibility leads to $\lambda / 2=j \pi$ with $j \neq 0$, because $\lambda=0$ makes the denominator $u^{2} / 4-k_{y}^{2}=\lambda^{2}$ vanish and does not lead to a solution. For $\lambda / 2=j \pi$ we have
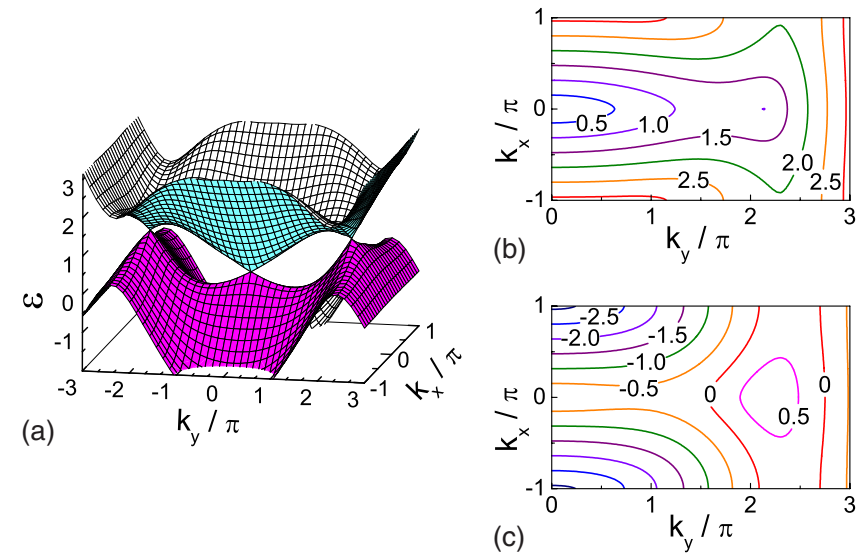

FIG. 3. (Color online) (a) Valence and conduction bands of the spectrum of a SL with barriers of width $W_{b}=0.4$ and height $u=6 \pi$. (b) and (c): projection contours of the conduction and valence band, respectively, on the $\left(k_{x}, k_{y}\right)$ plane. 

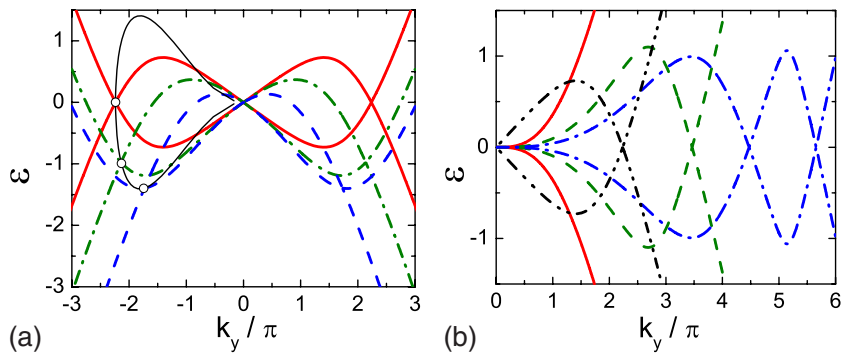

FIG. 4. (Color online) Slices of the SL spectrum along $k_{y}$ with $k_{x}=0$ and $u=6 \pi$. (a) The solid red, dash-dotted green, and dashed blue curves correspond to barrier widths $W_{b}=0.5,0.6$, and 0.7 , respectively. The thin black line is the curve on which the extra Dirac point, to the left of the main one at $k_{y}=0$, is located for various $W_{b}$. Only the new points to the left of the main one are shown. (b) As in (a) for fixed $W_{b}=0.5$. The solid red, dot-dot-dashed black, dashed green, and dash-dotted blue curves are for different values of the barrier height such that $u / 2=2 \pi, 3 \pi, 4 \pi$, and $6 \pi$, respectively.

$$
k_{y_{j, \pm}}= \pm \sqrt{\frac{u^{2}}{4}-4 j^{2} \pi^{2}}= \pm \sqrt{\left(\frac{V_{0}}{2 \hbar v_{F}}\right)^{2}-\left(\frac{2 j \pi}{L}\right)^{2}},
$$

where we reinserted the dimensions after the second equality sign. As such, Eq. (10) describes the spatial arrangement of the extra Dirac points along the $k_{y}$ axis. Also, it clearly shows how many points we have at particular values of $u$, namely, $2 \times(u \bmod 4 \pi)$, and where they are located in $\mathbf{k}$ space. Each time $u$ becomes a multiple of $4 \pi$ a new pair of Dirac points is generated for $k_{y}=0$. The condition $j \neq 0$ gives us a threshold value of $u=4 \pi$ for the emergence of the first pair. The integer $j$ denotes the $j$ th extra Dirac point, so the outer extra Dirac points have $j=1$ as they are generated first.

In Fig. 4(a) we show slices of the SL spectrum along $k_{y}$ for $k_{x}=0$ and $u=6 \pi$. The solid red, dash-dotted green, and dashed blue dashed curves correspond to barrier widths $W_{b}$ $=0.5,0.6$, and 0.7 respectively. The thin black line is the curve on which the extra Dirac points, on the left of the main one at $k_{y}=0$, are located for various $W_{b}$. In Fig. 4(b) we show slices of the spectrum along $k_{y}$ for $k_{x}=0$. The solid red, dotdot-dashed black, dashed green, and dash-dotted blue curves are for different values of the barrier height such that $u / 2=2 \pi, 3 \pi, 4 \pi$, and $6 \pi$ respectively. For values of $u / 2$ which are multiples of $2 \pi$, new Dirac points are generated. Interestingly, if new extra points are to arise, the dispersion becomes almost flat along the $k_{y}$ axis at the Dirac point, i.e., collimation occurs. We will come back to this issue in Sec. III C.

Unequal well and barrier widths. We return to the more general case of unequal well and barrier widths for which $W_{b} \neq 1 / 2$. It is more difficult to locate the extra Dirac points which no longer occur at the Fermi level as seen from the green and blue curves in Fig. 4(a) showing slices of spectra from Eq. (7) for $k_{x}=0$. By means of the symmetry $\varepsilon \rightarrow-\varepsilon$ for $W_{b} \leftrightarrow W_{w}$, we know the complementary plots for $W_{b} \rightarrow 1-W_{b}$. As can be seen, the extra Dirac points shift mainly down (up) in energy as $W_{b}$ increases (decreases). To find their coordinates $\left(\varepsilon, k_{x}=0, k_{y}\right)$ we assume $\sin \left(\lambda W_{w}\right)$ $=\sin \left(\Lambda W_{b}\right)=0$ and $\cos \left(\lambda W_{w}\right)=\cos \left(\Lambda W_{b}\right)= \pm 1 .{ }^{16}$ This gives (Appendix B)

$$
\begin{gathered}
\varepsilon_{j, m}=\frac{u}{2}\left(1-2 W_{b}\right)+\frac{\pi^{2}}{2 u}\left(\frac{j^{2}}{W_{w}^{2}}-\frac{(j+2 m)^{2}}{W_{b}^{2}}\right), \\
k_{y_{j, m}}= \pm\left[\left(\varepsilon_{j, m}+u W_{b}\right)^{2}-\left(j \pi / W_{w}\right)^{2}\right]^{1 / 2},
\end{gathered}
$$

where $j$ and $m$ are integers. This method also shows higher and lower crossing points if $m \neq 0$. In Fig. 4(a) the extra Dirac points on the left, obtained with this method, are indicated by open circles and the thin black curve shows their trajectory in $\left(E, k_{y}\right)$ space as the width $W_{b}$ varies. For a particular $u$ there is a minimal width $W_{b}$ (and a corresponding maximal width $W_{b} \rightarrow 1-W_{b}$ ) below (above) which the various extra Dirac points disappear. In Fig. 4(a) the "Dirac cones" at these crossing points for $m=0$ are not only reshaped with a renormalized anisotropic velocity but, as shown by the blue dashed curve, the "extra Dirac point" is not at a local minimum (maximum) of the conduction (valence) band.

\section{B. Analytical expression for the spectrum for small energies $\varepsilon$}

As the purpose is to have a closer look at the behavior of the extra Dirac points and we cannot prohibit $k_{y}$ from being large, we expand Eq. (8) for small energies, up to second order in $\varepsilon$, and obtain the following explicit dispersion relation:

$$
\varepsilon_{ \pm}= \pm\left[\frac{4\left|a^{2}\right|^{2}\left[k_{y}^{2} \sin ^{2}(a / 2)+a^{2} \sin ^{2}\left(k_{x} / 2\right)\right]}{k_{y}^{4} a \sin a+a^{2} u^{4} / 16-2 k_{y}^{2} u^{2} \sin ^{2}(a / 2)}\right]^{1 / 2},
$$

with $a=\left[u^{2} / 4-k_{y}^{2}\right]^{1 / 2}$.

If we only need the behavior of the spectrum near the $\mathrm{K}$ point (for small $k_{x}$ and $k_{y}$ ), it suffices to make an expansion for small $\varepsilon$ and $k_{y}$ in Eq. (8), up to third order in products of $\varepsilon$ and $k_{y}$ since this is the first order with an energy dependence. The result is

$$
2 \cos k_{x}-2+\varepsilon^{2}-k_{y}^{2} \sin ^{2}(u / 4) /(u / 4)^{2}=0 .
$$

Then we solve for the energy $\varepsilon$ and obtain

$$
\varepsilon \approx \pm\left[4 \sin ^{2} k_{x} / 2+k_{y}^{2} \sin ^{2}(u / 4) /(u / 4)^{2}\right]^{1 / 2} .
$$

In Fig. 5(a) we show $\varepsilon$ from Eq. (12) and compare it with the exact dispersion relation, for $k_{x}=0$, following from $\mathrm{Eq}$. (8). The expansion (12) is rather good for low energies near the extra Dirac points; accordingly, we will use Eq. (12) to further assess their behavior.

\section{Anisotropic velocity renormalization at the (extra) Dirac point(s)}

The spectrum in the low-energy range consists of two kinds of valleys, one near the main Dirac point and the other near the extra Dirac points. Near the original Dirac point the spectrum is almost linear, perpendicular to the barriers, and zero parallel to them, whereas near the extra Dirac points the 

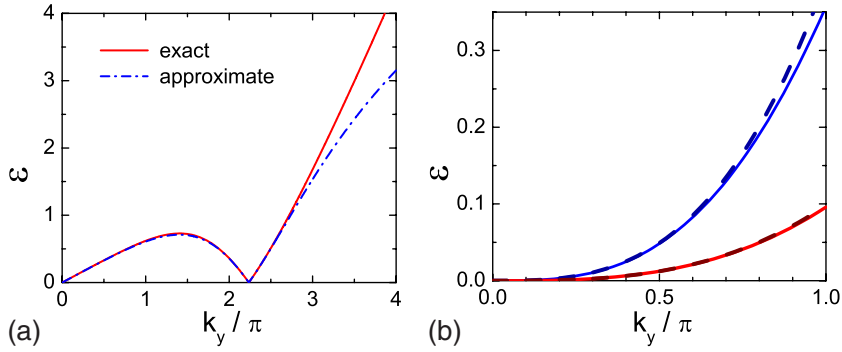

FIG. 5. (Color online) (a) Plot of the dispersion relation resulting from Eq. (12) (dashed blue curve) versus the exact one obtained from Eq. (8) (solid red curve) for $k_{x}=0$ and $u=6 \pi$. (b) The solid blue and red curves show, respectively, the zoomed-in plots of the solid red and dashed green curves of Fig. 4(b), i.e., for $u=4 \pi$ and $u=8 \pi$, respectively, and $W_{b}=W_{w}=1 / 2$. The approximation of these curves by Eq. (17) are the dashed curves.

situation can be reversed depending on the height of the barriers.

Group velocity along the $x$-axis at the Dirac point. To compare with the collimation found by Park et al. ${ }^{8}$ we notice that in Fig. 4(b), for the solid red, dashed green, and dash-dotted blue curves, corresponding to barrier heights which are multiples of $4 \pi$, the dispersion becomes more flat for small $k_{y}$. From Eq. (14) we could already expect that, to order $k_{y}^{2}$, the $k_{y}$ dependence disappears for these values of $u$. Further, if we expand Eq. (12) in powers of $k_{y}$ we obtain

$$
\begin{aligned}
\varepsilon= & \sin (u / 4) /(u / 4) k_{y}-\left(2 / u^{5}\right) \\
& \times\left[u^{3} \cos (u / 4)+4 u^{2} \sin (u / 4)-128 \sin ^{3}(u / 4)\right] k_{y}^{3}+O\left(k_{y}^{5}\right),
\end{aligned}
$$

which is linear in $k_{y}$, for small $k_{y}$, and the velocity becomes

$$
v_{y} / v_{F}=\partial \varepsilon / \partial k_{y} \approx \sin (u / 4) /(u / 4) .
$$

In Fig. 6 the velocities of the Dirac point, in the $x$ and $y$ directions, are given by the $j=0$ curves. For $u / 2=2 j \pi$ we have

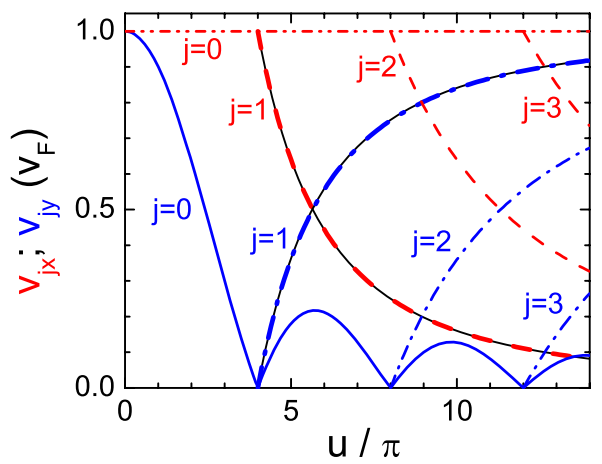

FIG. 6. (Color online) Velocities $v_{0 x}$ and $v_{0 y}$ (dash-dot-dotted red and solid blue curves, respectively), vs $u$ at the original Dirac point and $v_{j x}$ and $v_{j y}$ (dashed red and dash-dotted blue curves, respectively), given by Eqs. (19) and (21), vs $u$ at the extra Dirac points $j=1,2,3$. The thin black curves for the $j=1$ Dirac point, are obtained numerically from Eq. (8).

$$
\varepsilon \approx \pm k_{y}^{3} / 8 j^{2} \pi^{2}+O\left(k_{y}^{5}\right),
$$

which is cubic in $k_{y}$ for small $k_{y}$. If $j$ and consequently $u$ become larger the dispersion gets flatter. In Fig. 5(b) we plot Eq. (17) for $u=4 \pi$ and $u=8 \pi$ as dashed curves, which correspond, respectively, to the zoomed-in plots of the solid red and dashed green curves of Fig. 4(b), shown here as blue and red curves.

Group velocity along the y-axis at the extra Dirac points. The dispersion relation (12) for the $k_{y}$ values of the extra Dirac points, determined by $k_{y_{j \pm}}= \pm\left[u^{2} / 4-(2 j \pi)^{2}\right]^{1 / 2}$, gives us an idea of how dispersionless the spectrum near these points is along the $x$-direction. If $k_{y_{j, \pm}}$ exists, Eq. (12) becomes

$$
\varepsilon \approx 32 \pi^{2} j^{2} \sin \left(\left|k_{x}\right| / 2\right) / u^{2},
$$

and the partial derivative ${ }^{16}$ of $\varepsilon$ with respect to $k_{x}$ is

$$
v_{x} / v_{F}=\partial \varepsilon / \partial k_{x} \approx \operatorname{sgn}\left(k_{x}\right) 16 \pi^{2} j^{2} \cos \left(k_{x} / 2\right) / u^{2} .
$$

This means that for smaller $j$ (the most distant extra Dirac points) the group velocity along the $x$ direction is strongly suppressed. Further, as $u>4 j \pi$ must hold in order for $k_{y_{j, \pm}}$ to be real, $\left|v_{x}\right|$ is smaller than $1\left(\equiv v_{F}\right)$ at $k_{x}=0$. Only for the special values $u=4 j \pi$, for which new Dirac points appear, we have $\left|v_{j x}\right|=1$.

Meanwhile the dispersion in the $k_{y}$ direction is also of interest. First, let us take $k_{x}=0$ and expand the dispersion relation (12) for $k_{y}-k_{y_{j, \pm}} \ll 1$. To first order in this difference we obtain

$$
\varepsilon \approx \pm\left[4 k_{y_{j, \pm}}^{2} / u^{2}\right]\left(k_{y}-k_{y_{j, \pm}}\right) .
$$

This gives the velocity $v_{y}$ at the extra Dirac points

$$
\frac{v_{y}}{v_{F}}=\partial \varepsilon / \partial k_{y} \approx 4 k_{y_{j, \pm}}^{2} / u^{2}=4\left[u^{2} / 4-4 j^{2} \pi^{2}\right] / u^{2} .
$$

Since the coordinates of the extra Dirac points should be real, $k_{y_{j_{+}}}^{2}$ is positive and smaller than $u^{2} / 4$ and we have $v_{y}<1$ (the outer Dirac points, for $j=1$, show the largest $v_{y}$ ). This entails that both $v_{x}$ and $v_{y}$ are renormalized at the new Dirac points. A plot of the velocities of the extra Dirac points, in the $x$ and $y$ directions, given by Eqs. (19) and (21), is shown in Fig. 6. As seen, for the extra Dirac points, $v_{j x}$, shown by the dashed red curves, starts from $v_{F}$ and decreases to zero with increasing $u$ while $v_{j y}$ (dash-dotted blue curves), starts from zero and approaches $v_{F}$ slowly for large $u$. The thin solid black curves show the velocities for $j=1$, obtained numerically from Eq. (8). As can be seen, the two curves match almost perfectly.

\section{Density of states and conductivity}

Density of states. At zero temperature the density of states (DOS) $\mathrm{D}(\mathrm{E})$ is given by

$$
D(E)=\sum_{n, \mathbf{k}} \delta\left(E-E_{n \mathbf{k}}\right),
$$

with $E$ the energy. We show the DOS in Fig. 7, for $W_{b}=0.5$ (solid red curve) and $W_{b}=0.4$ (dashed blue curve), 


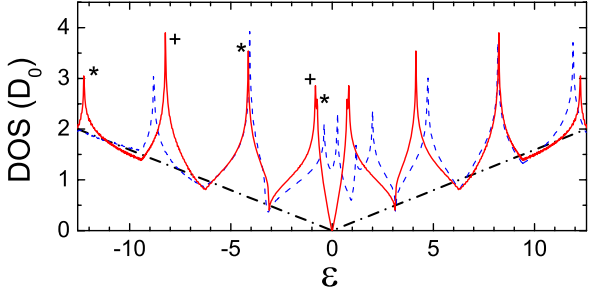

FIG. 7. (Color online) The DOS, for $u=6 \pi$, and $W_{b}=0.5$ and 0.4 is shown by, respectively, the solid red and dashed blue curve. Stars and crosses placed near the peaks of the solid red curve (for negative energies) are ascribed, respectively, to saddle points in the spectrum, for $k_{x}=0$, and to minima for $k_{x}=\pi$. The DOS without a SL potential is shown by the dash-dotted black curve.

as well as the DOS for graphene without any SL potential (dash-dotted black curve); the latter is given by $D(\varepsilon)=\varepsilon D_{0} / 2 \pi$, with $D_{0}=L / \hbar v_{F}$ the amount of states per unit area and $L$ is the period of the SL. The DOS shows an oscillating behavior. The dips in it are located at the crossing points in the energy bands for $k_{y}=0(\varepsilon=n \pi)$, while the peaks marked by a star are ascribed to the saddle points between the crossing points for $k_{x}=0$ and to the minima of the energy bands at the edge of the Brillouin zone, $k_{x}= \pm \pi$, marked by a cross. For $W_{b}=0.4 \neq W_{w}$ the DOS (dashed blue curve) does not vanish at $\varepsilon=0$ nor is it symmetric about this energy.

Conductivity tensor $\sigma$. The diffusive de conductivity $\sigma_{\mu \nu}$ for the SL system can be readily calculated from the spectrum if we assume a nearly constant relaxation time $\tau\left(E_{F}\right) \equiv \tau_{F}$. It is given by ${ }^{17}$

$$
\sigma_{\mu \nu}\left(E_{F}\right)=\frac{e^{2} \beta \tau_{F}}{A} \sum_{n, \mathbf{k}} v_{n \mu} v_{n \nu} f_{n \mathbf{k}}\left(1-f_{n \mathbf{k}}\right),
$$

with $A$ the area of the system, $n$ the energy band index, $\mu, \nu=x, y$, and $f_{n \mathbf{k}}=1 /\left\{\exp \left[\beta\left(E_{F}-E_{n \mathbf{k}}\right)\right]+1\right\}$ the equilibrium Fermi-Dirac distribution function; $E_{F}$ is the Fermi energy and $\beta=1 / k_{B} T$.

In Figs. 8(a) and 8(b) we show, respectively, $\sigma_{x x}$ and $\sigma_{y y}$ for a SL with $u=6 \pi$, and the temperature dependence is given by $\beta=\hbar v_{F} / k_{B} T L=20$ (in dimensionless units). The solid red and dashed blue curves correspond to $W_{b}=0.5$ and $W_{b}=0.4$, respectively. The dash-dotted black curve shows the conductivity at zero temperature and in the absence of a SL potential, $\quad \sigma_{x x}=\sigma_{y y}=\varepsilon_{F} \sigma_{0} / 4 \pi$, with $\varepsilon_{F}=E_{F} L / \hbar v_{F}$ and $\sigma_{0}=e^{2} / \hbar$. Notice that $\sigma_{x x}$ is an oscillating function of the Fermi level and recovers a quasi linear behavior similar to that in graphene without a SL potential when $\varepsilon_{F}$ is well above the barrier height, as shown in the inset, i.e., with the $x$ axis displaced over the potential height or well depth, i.e., $u / 2=3 \pi$. On the average $\sigma_{y y}$ increases with $\varepsilon_{F}$ and approaches the result without a SL for large energies.

The oscillations in both $\sigma_{x x}$ and $\sigma_{y y}$ result from the motion of the Fermi level through the different SL minibands. Notice that for $W_{b}=0.4$ the conductivities are asymmetric with respect to electron and hole conduction. In both cases, $W_{b}=W_{w}=0.5$ and $W_{b}=0.4, \sigma_{x x}$ shows dips at $\varepsilon_{F}=n \pi$, where energy band crossings occur in the spectrum for $k_{y}=0$. In the former case the DOS has dips occurring at the same energy
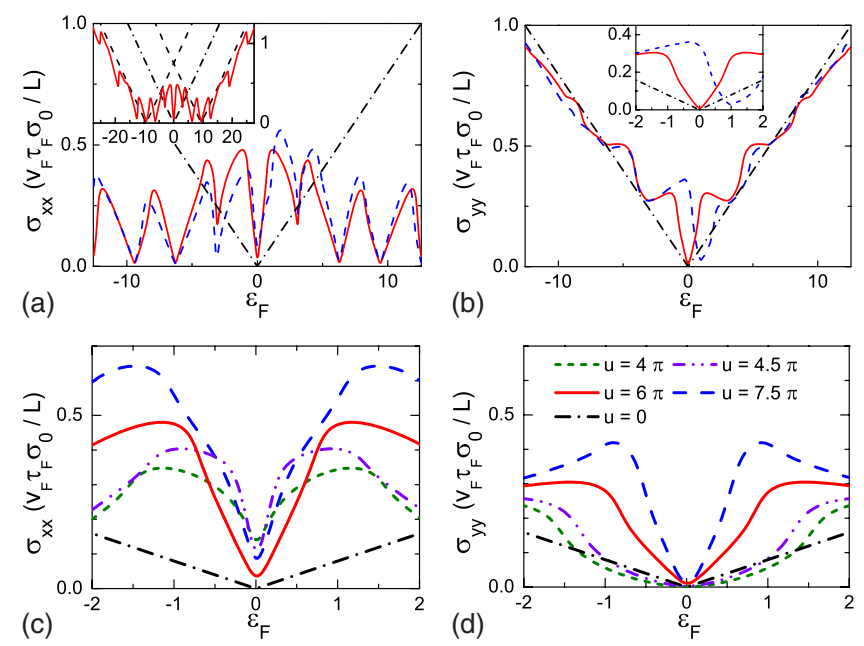

FIG. 8. (Color online) (a) and (b) show the conductivities $\sigma_{x x}$ and $\sigma_{y y}$ vs Fermi energy for a SL with $u=6 \pi$. The solid red and dashed blue curves are, respectively, for $W_{b}=0.5$ and $W_{b}=0.4$ $\left(W_{w}=1-W_{b}\right)$. The dash-dotted black curves show the conductivities in the absence of the SL potential, $\sigma_{x x}=\sigma_{y y}=\varepsilon_{F} \sigma_{0} / 4 \pi$. The inset in (a) shows the zoomed-out conductivity $\sigma_{x x}$, for $W_{b}=0.5$, and the dashed lines are the conductivities of graphene in the absence of a SL but with a constant nonzero potential applied, $-V_{0} / 2$ and $+V_{0} / 2$, so that $E_{F}$ is displaced by $V_{0} / 2$ and $u / 2=3 \pi$. The inset in (b) is a zoom on $\sigma_{y y}$ for small energies. (c) and (d) show $\sigma_{x x}$ and $\sigma_{y y}$, for $W_{b}=W_{w}=0.5$ for different potential heights, such that $u=4 \pi, 4.5 \pi$, $6 \pi, 7.5 \pi$, and small energies.

values that are dominated by the same crossings. In the latter case we see that, unlike the DOS, $\sigma_{x x}$ is almost unaffected by the extra Dirac points for low energies since the spectrum is almost flat near these points. Similarly, for $W_{b}=0.4$ we see that the minimum in $\sigma_{y y}$ is located at $\varepsilon_{F} \approx 1$, that is, the energy value for which the two extra Dirac points occur in the spectrum.

In Figs. 8(c) and 8(d) we show, respectively, $\sigma_{x x}$ and $\sigma_{y y}$, for a SL with $W_{b}=W_{w}=0.5$, for different potential heights, such that $u=0,4 \pi, 4.5 \pi, 6 \pi, 7.5 \pi$, and $\beta=\hbar v_{F} / k_{B} T L=20$. Notice that the conductivity $\sigma_{y y}$, in the low-energy range $\left(\varepsilon_{F}<1\right)$, is lower than that in the absence of a SL potential while its slope increases as the potential barriers become higher. This is due to the extra Dirac points that appear for larger potential heights, near which the velocity is larger along the $y$ axis. Notice that for $\varepsilon_{F}<1$ we have $\sigma_{x x}>\sigma_{y y}$ as a result of the inequality $v_{x}>v_{y}$ near the Dirac point.

\section{CONCLUSIONS}

We investigated the appearance of zero modes, touching points at the Fermi level (extra Dirac points) in the spectrum of single-layer graphene in the presence of a 1D superlattice (SL). The system was described by a Dirac-type Hamiltonian, and the SL barriers were square.

In the general case of unequal well and barrier widths, there is no particle-hole symmetry and the extra Dirac points are no longer located at the Fermi level. We obtained an analytical expression for the position of the crossing points in the spectrum. The extra "Dirac cones" that appear at the 
various crossing points are reshaped, i.e., they are no longer circular symmetric and the slope is renormalized. For fixed height of the barriers, we found lower and upper bounds for the barrier and well widths for the occurrence of these extra Dirac cones.

For a SL with equal well and barrier widths we complemented the investigations of Refs. 12 and 13, which numerically demonstrated the emergence of extra Dirac points (zero modes). In doing so we found a simple analytical expression for the spatial distribution of these points in $\mathbf{k}$ space as well as a threshold value of the potential strength for their appearance. Further, we approximated the dispersion relation for energies close to the Fermi energy and found an explicit expression for the $\mathbf{k}$ space behavior of the extra Dirac points at the Fermi level. Using this expression we showed how the group velocities at the various extra Dirac points are renormalized in the $x$ and $y$ directions. We also quantified how dispersionless the spectrum is in the neighborhood of a Dirac point along the $y$ direction and the emergence of new points at which the conduction and the valence bands touch each other.

Finally, we obtained numerically the density of states (DOS), which exhibits an interesting oscillatory behavior and is reflected in the conductivity of the system. We found that the dips in the DOS, for symmetric SLs, are located at the touching points in the spectrum for $\mathbf{k}=\mathbf{0}$, i.e., for $\varepsilon=n \pi$. For asymmetric SLs these dips persist but extra dips due to the extra Dirac points arise. The conductivity $\sigma_{x x}$ was found to have dips at the same values for $\varepsilon_{F}$ as the DOS while the main features of $\sigma_{y y}$ in the low-energy range are due to the spectrum near the extra Dirac points. We notice in passing that the influence of velocity renormalization on transport was not studied in Refs. 12 and 13 nor the modification of the extra Dirac points for unequal well and barrier widths.

\section{ACKNOWLEDGMENTS}

This work was supported by IMEC, the Flemish Science Foundation (FWO-Vl), the Belgian Science Policy (IAP), the Brazilian Council for Research $(\mathrm{CNPq})$, and the Canadian NSERC Grant No. OGP0121756.

\section{APPENDIX A: DISPERSION RELATION FOR PERIODIC SYSTEMS}

The wave functions in the regions before and after the barrier, labeled, respectively, by $j=1$ and $j=2$, can be written as $\psi_{j}(x)=\Omega_{j}(x) \mathcal{A}_{j}$, with

$$
\Omega=\left(\begin{array}{cc}
1 & 1 \\
s e^{i \phi} & -s e^{-i \phi}
\end{array}\right), \quad \mathcal{A}=\left(\begin{array}{l}
A \\
B
\end{array}\right) .
$$

Since the wave function of the entire periodic system is a Bloch function and the transfer matrix $\mathcal{T}$ connects the regions before and after the barriers, we have

$$
\psi(1)=e^{i k_{x}} \psi(0), \quad \mathcal{A}_{1}=\mathcal{T} \mathcal{A}_{2},
$$

with $k_{x}$ the Bloch wave vector. From these boundary conditions we extract the relation $\left(\lambda=\left[\varepsilon^{2}-k_{y}^{2}\right]^{1 / 2}\right)$

$$
e^{-i k_{x}} e^{i \lambda \sigma_{z}} \mathcal{A}_{2}=\mathcal{T} \mathcal{A}_{2}, \quad \text { with } \mathcal{T}=\left(\begin{array}{cc}
w & z \\
z^{*} & w^{*}
\end{array}\right)
$$

For nontrivial solutions of Eq. (A3), the determinant of $\mathcal{A}_{2}=(A, B)^{T}$ must be zero, i.e.,

$$
\operatorname{det}\left(\begin{array}{cc}
e^{-i k_{x}} e^{i \lambda}-w & -z \\
-z^{*} & e^{-i k_{x}} e^{-i \lambda}-w^{*}
\end{array}\right)=0 .
$$

Evaluating the determinant gives the dispersion relation

$$
\cos k_{x}=\Re\left\{w e^{-i \lambda}\right\}=\cos \left(\delta_{t}+\lambda\right) /|t|,
$$

with $1 / w=t=|t| e^{i \delta_{t}}$.

\section{APPENDIX B: CROSSING POINTS FOR UNEQUAL BARRIER AND WELL WIDTHS}

Suppose a solution $\left(\varepsilon, k_{x}=0, k_{y}\right)$ of the dispersion relation (7) is known for which the derivative $\partial \varepsilon / \partial k_{y}$ at a certain $k_{y}$ value is undefined; then this $k_{y}$ value can be a crossing point. The condition for such a solution is $\sin \left(\lambda W_{w}\right)=\sin \left(\Lambda W_{b}\right)$ $=0$, and $\cos \left(\lambda W_{w}\right)=\cos \left(\Lambda W_{b}\right)= \pm 1$, which entails

$$
\begin{gathered}
\lambda W_{w}=j \pi, \\
\Lambda W_{b}=(j+2 m) \pi,
\end{gathered}
$$

with $j$ and $m$ integers. Explicitly we obtain

$$
\begin{gathered}
{\left[\left(\varepsilon+u W_{b}\right)^{2}-k_{y}^{2}\right] W_{w}^{2}=(j \pi)^{2},} \\
\left\{\left[\varepsilon-u\left(1-W_{b}\right)\right]^{2}-k_{y}^{2}\right\} W_{b}^{2}=[(j+2 m) \pi]^{2} .
\end{gathered}
$$

Subtracting the second equation from the first one in Eq. (B2) gives

$$
2 u \varepsilon-u^{2}\left(1-2 W_{b}\right)=\pi^{2}\left(\frac{j^{2}}{W_{w}^{2}}-\frac{(j+2 m)^{2}}{W_{b}^{2}}\right),
$$

from which the corresponding value of the energy $\varepsilon$ can be extracted. Substituting this value in the first of Eqs. (B2) one obtains

$$
\begin{gathered}
\varepsilon_{j, m}=\frac{u}{2}\left(1-2 W_{b}\right)+\frac{\pi^{2}}{2 u}\left(\frac{j^{2}}{W_{w}^{2}}-\frac{(j+2 m)^{2}}{W_{b}^{2}}\right), \\
k_{y_{j, m}}= \pm\left[\left(\varepsilon_{j, m}+u W_{b}\right)^{2}-\left(j \pi / W_{w}\right)^{2}\right]^{1 / 2} .
\end{gathered}
$$


${ }^{1}$ K. S. Novoselov, A. K. Geim, S. V. Morozov, D. Jiang, Y. Zhang, S. V. Dubonos, I. V. Grigorieva, and A. A. Firsov, Science 306, 666 (2004).

${ }^{2}$ O. Klein, Z. Phys. 53, 157 (1929).

${ }^{3}$ M. I. Katsnelson, K. S. Novoselov, and A. K. Geim, Nat. Phys. 2, 620 (2006); J. M. Pereira, Jr., P. Vasilopoulos, and F. M. Peeters, Appl. Phys. Lett. 90, 132122 (2007).

${ }^{4}$ N. Stander, B. Huard, and D. Goldhaber-Gordon, Phys. Rev. Lett. 102, 026807 (2009); A. F. Young and P. Kim, Nat. Phys. 5, 222 (2009)

${ }^{5}$ J. Schliemann, D. Loss, and R. M. Westervelt, Phys. Rev. Lett. 94, 206801 (2005); W. Zawadzki, Phys. Rev. B 72, 085217 (2005); R. Winkler, U. Zulicke, and J. Bolte, ibid. 75, 205314 (2007).

${ }^{6}$ A. H. Castro Neto, F. Guinea, N. M. R. Peres, K. S. Novoselov, and A. K. Geim, Rev. Mod. Phys. 81, 109 (2009).

${ }^{7}$ C.-H. Park, L. Yang, Y.-W. Son, M. L. Cohen, and S. G. Louie, Nat. Phys. 4, 213 (2008).

${ }^{8}$ C.-H. Park, Y.-W. Son, L. Yang, M. L. Cohen, and S. G. Louie, Nano Lett. 8, 2920 (2008).

${ }^{9}$ Y. P. Bliokh, V. Freilikher, S. Savel'ev, and F. Nori, Phys. Rev. B 79, 075123 (2009).
${ }^{10}$ J. H. Ho, Y. H. Chiu, S. J. Tsai, and M. F. Lin, Phys. Rev. B 79, 115427 (2009).

${ }^{11}$ In Ref. 9 a nearest-neighbor, tight-binding method was applied with a large rectangular supercell along a sinusoidal potential as basis. Extra touching points at the Fermi level were observed and, together with the group velocity in their vicinity, their influence on the density of states was demonstrated.

${ }^{12}$ C.-H. Park, Y.-W. Son, L. Yang, M. L. Cohen, and S. G. Louie, Phys. Rev. Lett. 103, 046808 (2009).

${ }^{13}$ L. Brey and H. A. Fertig, Phys. Rev. Lett. 103, 046809 (2009).

${ }^{14}$ D. J. Griffiths and C. A. Steinke, Am. J. Phys. 69, 137 (2001).

${ }^{15}$ M. Barbier, F. M. Peeters, P. Vasilopoulos, and J. M. Pereira, Jr., Phys. Rev. B 77, 115446 (2008).

${ }^{16}$ The implicit function theorem cannot be applied if the gradient (Jacobian) of the dispersion relation is zero. This occurs because of the crossing of the bands at these points: from the form of the derivatives, too long to write down, we can see that they are both zero if $\sin \left(\lambda W_{w}\right)=\sin \left(\Lambda W_{b}\right)=0 \quad$ while $\cos \left(\lambda W_{w}\right)$ $=\cos \left(\Lambda W_{b}\right)$.

${ }^{17}$ M. Charbonneau, K. M. van Vliet, and P. Vasilopoulos, J. Math. Phys. 23, 318 (1982). 